\title{
Immunohistochemical Demonstration of GABAergic Neurons in the Enteric Nervous System
}

\author{
Kristján R. Jessen, Judith M. Hills, and M. Jill Saffrey \\ Department of Anatomy and Embryology, and Centre for Neuroscience, University College London, \\ London WC1E 6BT, England
}

\begin{abstract}
Application of a highly specific antiserum against GABA to whole-mount preparations of the guinea pig and rat myenteric plexus resulted in discrete and unambiguous immunolabeling of a subpopulation of myenteric neuronal cell bodies and fibers.

The anti-GABA antiserum, which was raised against GABA conjugated by glutaraldehyde to BSA, was applied to glutaraldehyde-fixed whole mounts and subsequently visualized using the peroxidase-antiperoxidase method. In the guinea pig ileum and colon, immunoreactive varicose nerve fibers and scattered nerve cell bodies were found within the myenteric plexus. Immunostained fibers were also seen in the tertiary plexus and in the circular muscle, running parallel to the muscle bundles. GABA immunoreactivity in these intramuscular nerves was most pronounced in the colon. In the rat, immunoreactive fibers were prominent throughout the myenteric plexus. They formed dense networks within the myenteric ganglia, which also contained immunopositive nerve cell bodies, and ran between them in the interconnecting nerves. Some immunoreactive nerve fibers were seen in the circular muscle. Control experiments using non-immune sera or adsorbed anti-GABA antiserum showed no staining.
\end{abstract}

These results add a definitive support to our previous suggestion that GABA serves as an autonomic neurotransmitter in vertebrates. In addition to the present immunohistochemical evidence, this hypothesis is supported by biochemical, autoradiographic, transmitter release, electrophysiological, and pharmacological studies on the enteric nervous system of several species. It is now important to determine the functional role of GABAergic neurons within the complex neuronal circuitry that controls gut functions.

Strong, but indirect, evidence for the presence of GABAergic neurons within the autonomic nervous system of vertebrates has accumulated in studies on the myenteric ganglia of the gastrointestinal tract. We here report the first direct demonstration of these cells, using a highly specific anti-GABA antibody.

The original biochemical and autoradiographic experiments on enteric GABAergic neurons were carried out on the myenteric plexus from the guinea pig cecum (Jessen et al., 1979). Myenteric ganglia, isolated from the gut wall to avoid the effects of contaminating tissue, were able to synthesize and accumulate significant quantities of ${ }^{3} \mathrm{H}-\mathrm{GABA}$ after incubation in ${ }^{3} \mathrm{H}$-glutamic acid, the main precursor of neuronal GABA synthesis. In homogenates of these ganglia, it was also possible to measure the specific activity of the enzyme responsible for conversion

\footnotetext{
Received July 10, 1985; revised Sept. 9, 1985; accepted Sept. 10, 1985.

We are grateful to Dr. P. Somogyi for the generous donation of anti-GABA antiserum. This work was supported by the Medical Research Council of Great Britain and the Wellcome Trust.

Correspondence should be addressed to K. R. Jessen, Department of Anatomy and Embryology, University College London, Gower Street, London WC1E 6BT, UK.

Copyright (C) 1986 Society for Neuroscience $0270-6474 / 86 / 061628-07 \$ 02.00 / 0$
}

of glutamic acid to GABA, glutamic acid decarboxylase (GAD; EC4.1.1.15). Gut muscle, containing the myenteric plexus as only a very small weight percentage, nevertheless was about $3 \times$ as rich in endogenous GABA as a control muscle not containing the plexus. A selective labeling of a subpopulation of neurons in the myenteric ganglia was obtained following incubation of gut segments in low concentrations of ${ }^{3} \mathrm{H}-\mathrm{GABA}$ and processing for light- and electron-microscopic autoradiography. Similarly, a highly selective labeling of neurons carrying long neurites was seen in autoradiographic studies on myenteric ganglia maintained in culture (Jessen, 1981; Jessen et al., 1979, 1983).

Many other studies now support these data. Autoradiographic labeling of certain myenteric neurons on incubation in ${ }^{3} \mathrm{H}-\mathrm{GABA}$ has been confirmed using whole mounts of the guinea pig ileum (Krantis and Kerr, 1981), and the presence of such cells has been shown in other areas of the gut and in different species, using cultured enteric plexuses from the rat, guinea pig, and chick (Saffrey et al., 1983). In a layer of cat colon containing the myenteric plexus endogenous, GABA levels and specific GAD activity are, respectively, about 20 and $10 \times$ higher than in surrounding muscle(Miki et al., 1983; Taniyama etal., 1982b). Release of exogenous ${ }^{3} \mathrm{H}-\mathrm{GABA}$, which is TTX- sensitive and $\mathrm{Ca}^{2+}$-dependent, has been obtained on electrical stimulation of gut segments from the guinea pig and cat (Hills and Jessen, 1983; Jessen et al., 1983; Kerr and Krantis, 1983; Taniyama et al., 1982a). It has been proved that such release can take place from neurons that are intrinsic to the myenteric plexus, by demonstrating $\mathrm{Ca}^{2+}$-dependent release of exogenous GABA from cultured myenteric plexuses devoid of extrinsic innervation, using $\mathrm{K}^{+}$as a depolarizing agent (Jessen et al., 1983). Most recently, release of endogenous GABA from gut neurons has been shown in the cat and guinea pig (Tanaka and Taniyama, 1985; Taniyama et al., 1983).

In the present study we have been able to visualize directly a distinct population of myenteric neurons and their projections in whole mounts of the myenteric plexus from the rat and guinea pig using a highly specific anti-GABA antibody. This adds a conclusive support to our hypothesis that the enteric nervous system contains a population of GABAergic neurons. The role of these cells in the neuronal control of gut function now remains to be determined. Part of this work has already appeared in preliminary form (Hills et al., 1985).

\section{Materials and Methods}

\section{Tissue preparation}

Three- to six-d-old (total of 4 experiments) and adult (total of 20 experiments) guinea pigs, and 3-week to 2-month-old rats (total of 15 experiments) were killed by a blow on the neck and bled. Following removal of the guinea pig ileum and colon and rat colon, whole-mount preparations (see below) were in some experiments made and fixed (see below) without delay, while in other experiments, the tissues were placed in colchicine $(10 \mathrm{mg} / 1000 \mathrm{ml})$ containing Krebs' solution of the follow- 

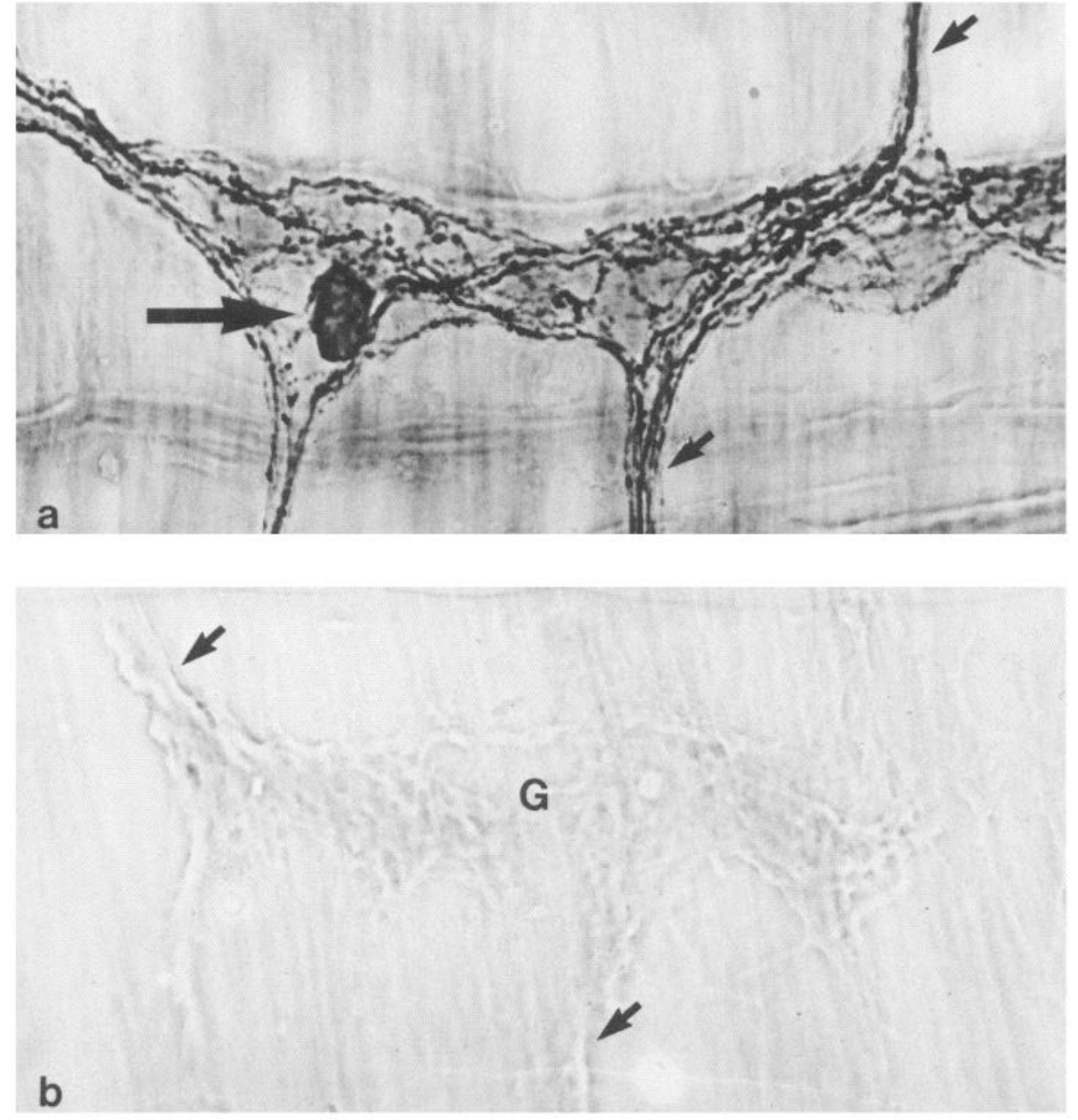

Figure 1. a, Immunoperoxidase micrograph of the rat colon, treated with anti-GABA antibodies. The micrograph shows a single oblong ganglion and three interconnecting nerve strands (two of which are labeled with small arrows). Within the ganglion, an immunopositive neuronal cell body (long arrow) can be seen, as well as numerous immunolabeled nerve fibers, lying among unlabeled neurons and glia. Immunopositive nerve fibers can also be seen in the interconnecting strands. A bundle of anti-GABA-labeled axons appears to traverse the ganglion, running from one of the arrowed interconnecting strands to the other. $b$, Immunoperoxidase micrograph of the rat colon, treated with adsorbed anti-GABA antiserum. A single oblong ganglion $(G)$, with associated interconnecting strands (examples labeled with arrows), occupies the center of the field. No immunopositive structures are present. $\times 500$. ing composition (mM): $\mathrm{NaCl} 133.0 ; \mathrm{KCl} 4.7 ; \mathrm{NaH}_{2} \mathrm{PO}_{4} 1.3 ; \mathrm{NaHCO}_{3}$ $16.3 ; \mathrm{MgSO}_{4} 0.6 ; \mathrm{CaCl}_{2} 2.5$; glucose 7.7. The Krebs' solution was continuously gassed with $95 \% \mathrm{O}_{2} / 5 \% \mathrm{CO}_{2}$ and maintained at $37^{\circ} \mathrm{C}$. After $3 \mathrm{hr}$, longitudinal muscle-myenteric plexus whole-mount preparations were made and pinned onto Silgard slightly stretched. All the tissues were fixed in $5 \%$ glutaraldehyde in PBS for 4-5 hr, after which time they were washed in PBS for 30 min prior to being left overnight at $4^{\circ} \mathrm{C}$ in PBS. The immunostaining was somewhat stronger in the colchicinetreated preparations compared to those fixed without colchicine treatment. A comparable observation was made by Somogyi et al. (1985).

\section{Antisera}

The antiserum to GABA (code no. GABA7), which was raised in rabbits and recognizes a condensation product of GABA and glutaraldehyde, has been extensively characterized (Hodgson et al., 1985; Somogyi and Hodgson, 1985; Somogyi et al., 1985). The antiserum was used at a dilution of $1: 2000$ to $1: 5000$.

\section{Immunohistochemical procedure}

The entire immunohistochemical procedure was carried out at room temperature. After fixation the whole mounts were dehydrated in 80 , 95 , and $2 \times 100 \%$ ethanol for $30 \mathrm{~min}$ each. Endogenous peroxidase was inactivated with $1 \% \mathrm{H}_{2} \mathrm{O}_{2}$ in methanol for $10 \mathrm{~min}$, and the preparations were subsequently cleared and rehydrated according to the following procedure: $2 \times 100 \%$ ethanol, Histoclear (National Diagnostics), 100, 80 , and $50 \%$ ethanol, each for $30 \mathrm{~min}$. The preparations were then washed in PBS for $60 \mathrm{~min}$. The tissues were preincubated in antibody diluting solution (ABDS) of the following composition (mM): $\mathrm{NaCl} 300$, Tris/ $\mathrm{HCl} 100, \mathrm{Na} /$ azide 200 , Triton X-100 $0.25 \%$, and gelatin (60 Bloom) $1 \%$, containing $3 \%$ normal sheep serum for $30 \mathrm{~min}$. Incubation in the GABA antiserum took place overnight. After a 45 min wash in ABDS, sheep anti-rabbit (Miles Laboratories) antiserum diluted 1:10 with ABDS was applied for $60 \mathrm{~min}$. Following a further wash, the preparations were incubated in rabbit peroxidase-antiperoxidase (Dakopatts $\mathrm{A} / \mathrm{S}$ ) diluted 1:70 with ABDS for 90 min. After washes in ABDS (30 min), PBS (10 $\mathrm{min})$, and $50 \mathrm{~mm}$ Tris $(10 \mathrm{~min})$, the tissues were preincubated for 10 min in 3,3'-diaminobenzidine tetrahydrochloride (Sigma Chemical Co.), $25-50 \mathrm{mg} / 100 \mathrm{ml}$, made up in $50 \mathrm{~mm}$ Tris, $\mathrm{pH} \mathrm{7.2,} \mathrm{followed} \mathrm{by} \mathrm{reaction}$ for $10-20 \mathrm{~min}$ in a similar solution now containing $0.01 \%$ hydrogen peroxide. The tissues were then washed in PBS for $30 \mathrm{~min}$ and incubated in $0.1 \%$ osmium tetroxide for $30 \mathrm{sec}$ to enhance contrast. After a wash in PBS, the preparations were dehydrated, cleared, and mounted in D.P.X. (Raymond Lamb).

\section{Specificity of the immunohistochemical procedure}

Specificity was tested by replacing the primary antiserum with either nonimmune rabbit serum at the same dilution as the anti-GABA antiserum or adsorbed anti-GABA antiserum. To obtain the adsorbed serum the diluted antiserum was subjected to solid-phase adsorption, using the antigen coupled to polyacrylamide beads, essentially as described by Somogyi et al. (1985).

\section{Results}

Immunostaining with anti-GABA antibodies was studied in whole mounts of the myenteric plexus from the adult guinea pig ileum and colon, newborn guinea pig colon, and adult rat colon. In these preparations the plexus lies as a two-dimensional network on top of the outermost muscle coat of the gut wall, the longitudinal muscle. The plexus is in vivo sandwiched between this layer and the major muscle layer of the gut, the circular muscle. Although most of this layer is stripped off in the whole mounts, thin bands of circular muscle sometimes remain, lying over the myenteric plexus. These preparations can 


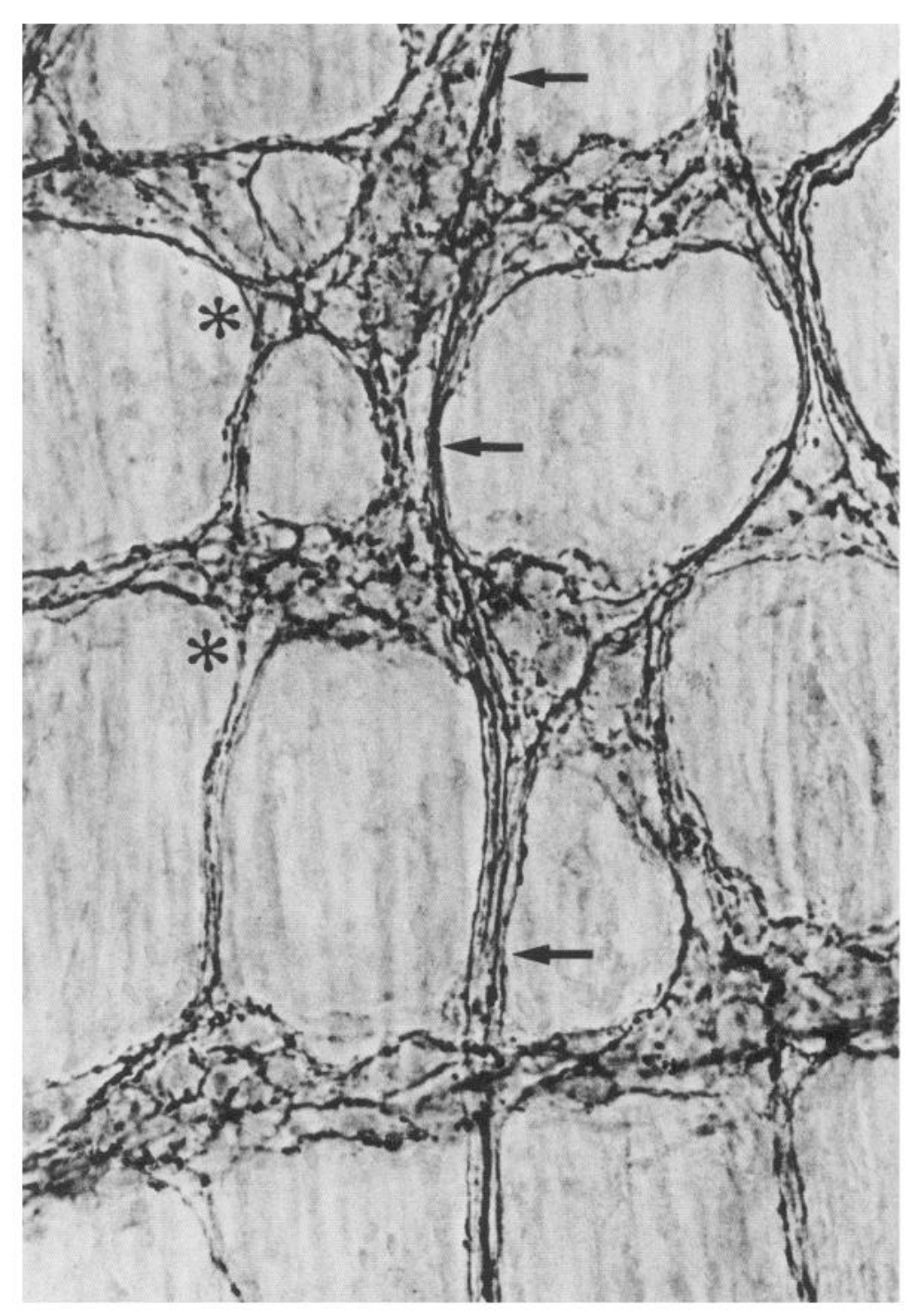

Figure 2. Immunoperoxidase micrograph of the rat colon treated with anti-GABA antibodies. The micrograph, which shows three ganglia running transversely across the field together with interconnecting nerve strands, illustrates the general appearance of GABA-labeled nerve fibers within the rat myenteric plexus. Fairly dense network of strongly immunopositive fibers is present within all of the ganglia, and labeled fibers run in most of the interconnecting strands. At the junctions between interconnectives and ganglia, the immunopositive fibers of the strand either merge with the fiber network of the ganglion (e.g., at asterisks) or form bundles running across the ganglia (see Fig. 3). Such bundles can often be followed through more than one ganglion (e.g., the arrowed bundle). $\times 400$. therefore be used to study (1) nerve fibers and cell bodies within the ganglia and interconnecting strands that together constitute the network of the primary plexus; (2) the secondary and tertiary plexuses, i.e., the finer fiber network that lies within and is continuous with the meshwork of the primary plexus, and in turn gives rise to fibers projecting to the circular muscle; and (3) the innervation of that part of the circular muscle that lies relatively near the myenteric plexus.

In all four types of preparation studied, the application of anti-GABA antibodies resulted in strong immunostaining of nerve fibers within the primary plexus and circular muscle, and of neuronal cell bodies scattered in the myenteric ganglia. No immunostaining was seen in parallel experiments using adsorbed anti-GABA antibodies or non-immune serum.

In the rat colon, immunoreactive fibers were very prominent in the myenteric plexus, in both the interconnecting strands and also surrounding neuronal cell bodies within the ganglia, some of which were also immunopositive (Figs. 1, 2). The GABApositive neuronal perikarya showed quite variable staining intensity, and they were often found near the edge of the ganglia. On entering a ganglion via an interconnecting strand, a bundle of immunoreactive fibers often branched among the cells of the ganglion (Fig. 2). Alternatively, they formed quite prominent pathways across the ganglion to exit from it via another inter- connecting strand (Figs. 2, 3). This pattern was often repeated through a chain of several ganglia, lying along an axis parallel to the longitudinal muscle. This suggests the presence of long pathways of GABA-containing axons running along the oralaboral axis of the gut within the myenteric plexus. The abundance of immunostained fibers within the myenteric plexus made it difficult to trace individual axons with certainty for more than a few hundred microns at most. In addition to the dense network of fibers within the myenteric plexus, immunoreactive fibers could also be seen in the circular muscle, running parallel to the long axis of the muscle bundles (Fig. 4).

In the guinea pig colon and ileum the density of immunostained fibers within the myenteric plexus was lower than that seen in the rat. As in the rat, immunoreactive neuronal cell bodies, showing marked variation in reaction intensity, were scattered within the ganglia, often in a peripheral location. Individual nerve fibers, which were more clearly beaded or varicose than in the rat, could therefore be more easily followed, running between, and within, ganglia (Fig. $5 a, b$ ). In addition, a fine network of immunoreactive fibers was present in the secondary and tertiary plexuses (Fig. $5 c, d$ ). Furthermore, immunostained fibers were prominent in the circular muscle (Figs. $5 d, 6)$. These fibers mostly ran in parallel with the long axis of the muscle bundles, while some lateral branching was also noted. 

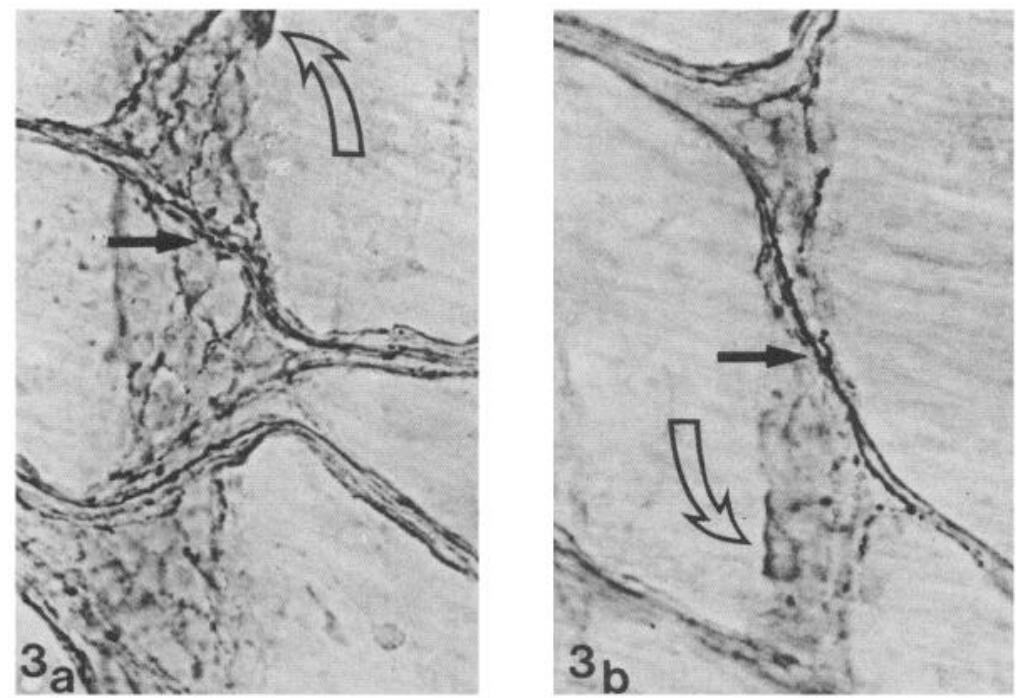

Figure 3. Immunoperoxidase micrographs of the rat colon treated with anti-GABA antibodies. These micrographs illustrate apparent pathways formed by GABA-immunoreactive fibers across myenteric ganglia, running approximately parallel to the longitudinal axis of the colon. $a$, The upper one of two axon bundles traversing the ganglion is indicated (solid straight arrow). An immunopositive neuronal cell body (open curved arrow) is also present lying slightly out of the plane of focus. $b$, Traversing axon bundle (solid straight arrow) lies on top of a ganglion. An immunolabeled neuronal cell body (open curved arrow) and nerve fibers within the ganglion lie well out of the plane of focus. $\times 400$.

The immunoreactivity in these fibers was considerably heavier than that seen in fibers within the plexus. This could be due to a higher concentration of GABA per fiber, larger fibers, or possibly to several GABA-containing axons running in close proximity to each other. No clear difference was noted in the pattern of immunoreactivity between the adult and newborn guinea pig colon.

\section{Discussion}

The most important part of the present results is the observation that a highly specific anti-GABA antibody selectively reacted with a subpopulation of neurons and nerve fibers in the enteric nervous system. This adds definitive support to our hypothesis that the enteric nervous system contains a population of GABAergic neurons.

The demonstration of immunoreactive neuronal cell bodies within the myenteric ganglia shows that GABAergic neurons are intrinsic to the myenteric plexus. This was previously indicated in autoradiographic experiments (Jessen et al., 1979, 1983; Krantis and Kerr, 1981; Saffrey et al., 1983) and in studies on the neuronal release of exogenous ${ }^{3} \mathrm{H}-\mathrm{GABA}$ from myenteric plexus cultures that lack extrinsic innervation (Jessen et al., 1983). It remains to be determined what percentage of the myenteric neurons in the ileum and colon are GABAergic. The only estimate of the frequency of these cells comes from the guinea pig colon. In this tissue, autoradiographic studies indicate that approximately $5 \%$ of the myenteric neurons possessed highaffinity uptake sites for ${ }^{3} \mathrm{H}-\mathrm{GABA}$ and were thus likely to be GABAergic (Jessen et al., 1979).

It is now clear that the enteric plexuses contain a number of different neuronal subpopulations. These can be characterized by morphological criteria (Cook and Burnstock, 1976) or by their content of neurotransmitters or neurotransmitter candidates, which are sometimes found in coexistence with one another
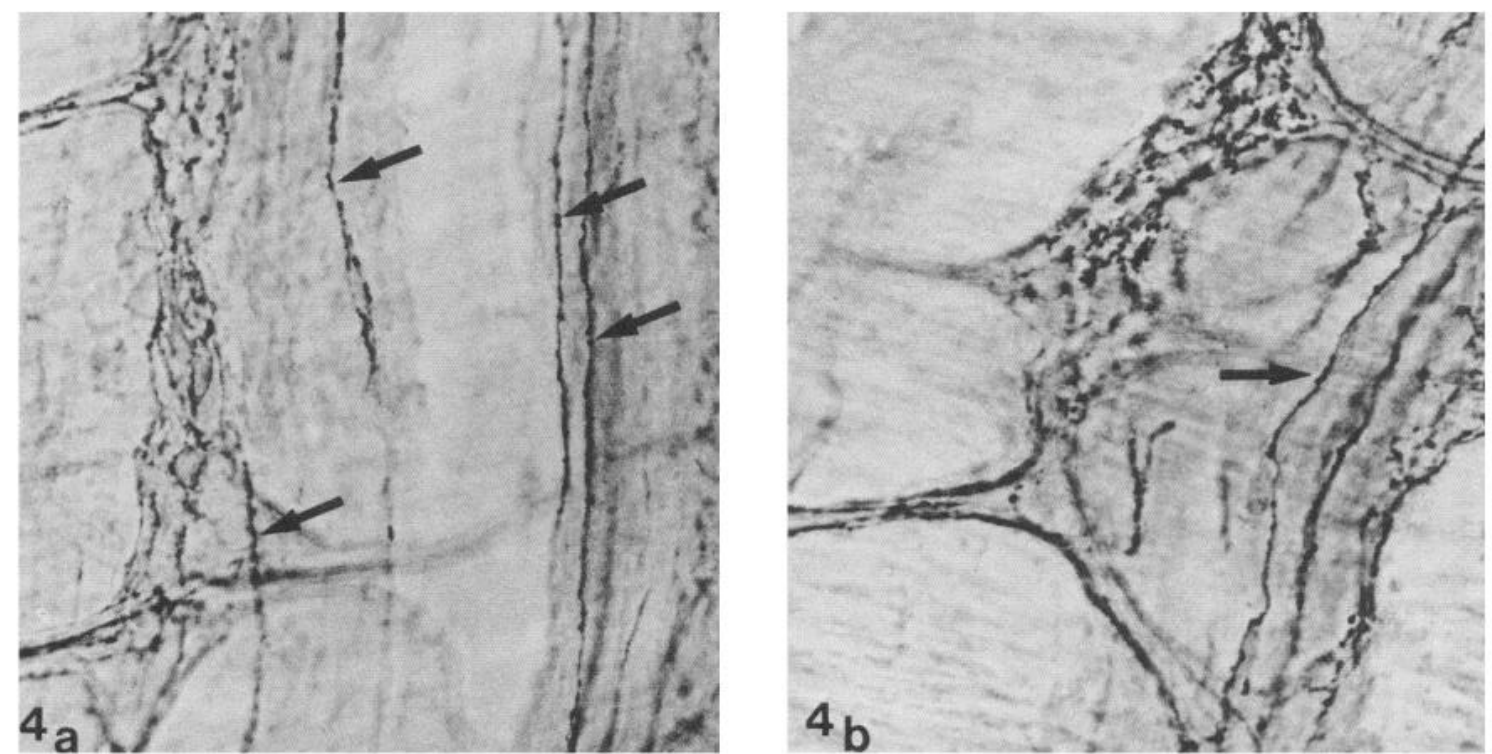

Figure 4. Immunoperoxidase micrograph of the rat colon treated with anti-GABA antibodies. These micrographs show GABA-immunoreactive fibers outside the myenteric plexus, in the circular muscle layer. The fibers (examples labeled with arrows) are present in bands of circular muscle that remained attached to the longitudinal muscle-myenteric plexus when the whole-mounts were prepared. The GABA-positive fibers run parallel to the long axis of the circular muscle bundles. In both micrographs, a myenteric ganglion can be seen, lying below the plane of focus, to the left of the arrowed fibers. $\times 400$. 


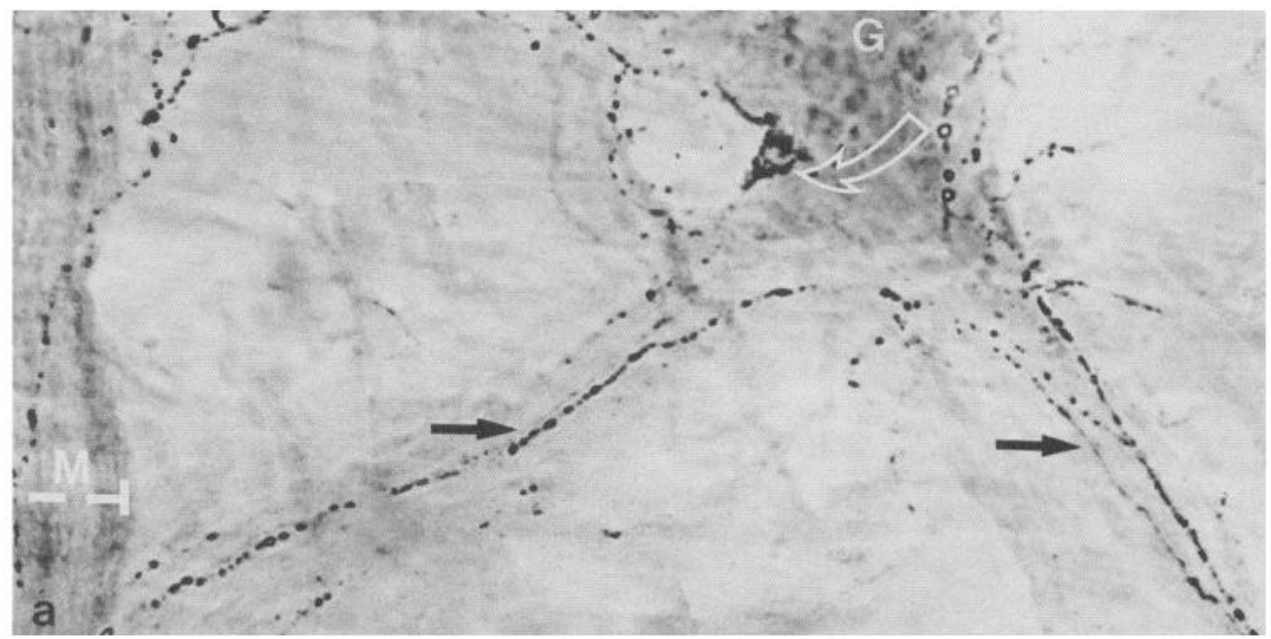

Figure 5. Immunoperoxidase micrographs of the guinea pig colon ( $a$, $b, d)$ and ileum $(c)$ treated with antiGABA antibodies. $a$, Single ganglion $(G)$ of the myenteric plexus is seen lying on top of the outer longitudinal muscle. It gives rise to two interconnecting strands (arrows). On the $e x$ treme left, a band of circular muscle $(M$, its extent indicated by a stippled line) has remained attached to the preparation. Note the presence of varicose, immunopositive nerve fibers, especially prominent in the interconnecting strands, and an immunolabeled nerve cell body near the edge of the ganglion (open curved arrow). The varicose fibers partly overlying the circular muscle to the left belong to the tertiary plexus. $b$, Two ganglia $(G)$ are linked by one interconnecting strand (double-headed arrow) and give rise to several others (single arrows). Varicose, GABA-positive nerve fibers run in the strands and are present inside the ganglia. In addition, several other labeled fibers (e.g., double arrows), generally lying out of the plane of focus, are seen within the meshwork of the primary plexus; these belong to the tertiary plexus. $c$, The fine meshwork of the tertiary plexus of the ileum can be seen; $d$, the tertiary plexus of the colon has the appearance of a delicate network of varicose fibers, underneath which lies a ganglion $(G)$ of the myenteric plexus. On the extreme right is a band of circular muscle $(M$, its width indicated by a stippled line) also containing GABA labeled fibers. These are coarser and straighter than those of the tertiary plexus. $\times 400$.
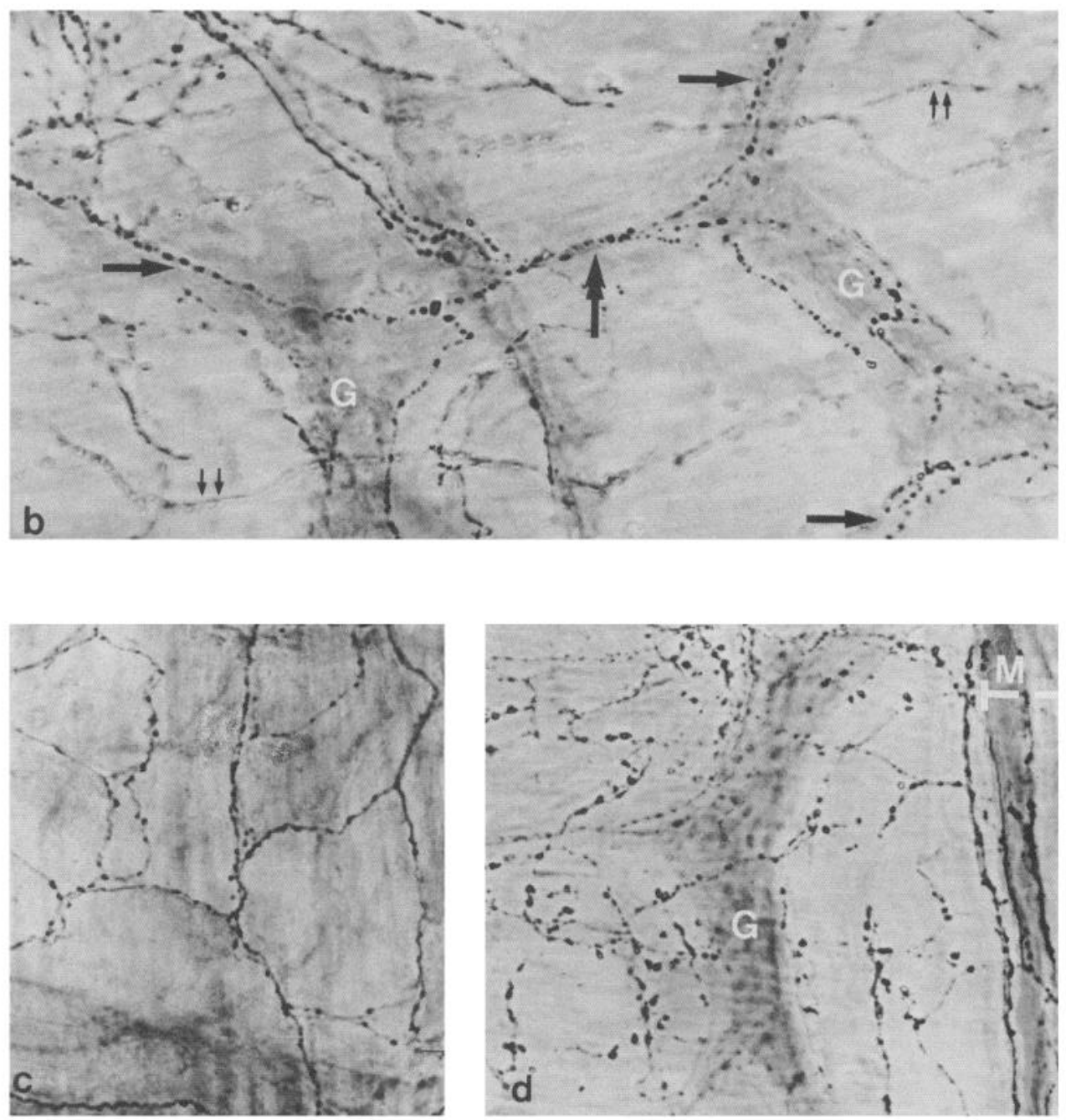

in single neurons (Furness and Costa, 1980; Furness et al., 1984; Jessen et al., 1980; Legay et al., 1984; Melander et al., 1985; Schultzberg et al., 1980; Wolter, 1985). Coexistence of GABA and other neuronal population markers-including serotonin, somatostatin, and colecystokinin - has been observed in central neurons (Pujol, 1984; Somogyi et al., 1984). All of these sub- stances are present among enteric neurons, and it is possible that the neuronal population visualized by the anti-GABA antibodies partly overlaps with previously described populations of myenteric neurons that contain these or other neuroactive molecules.

In addition to neuronal perikarya, nerve fibers were a prom- 

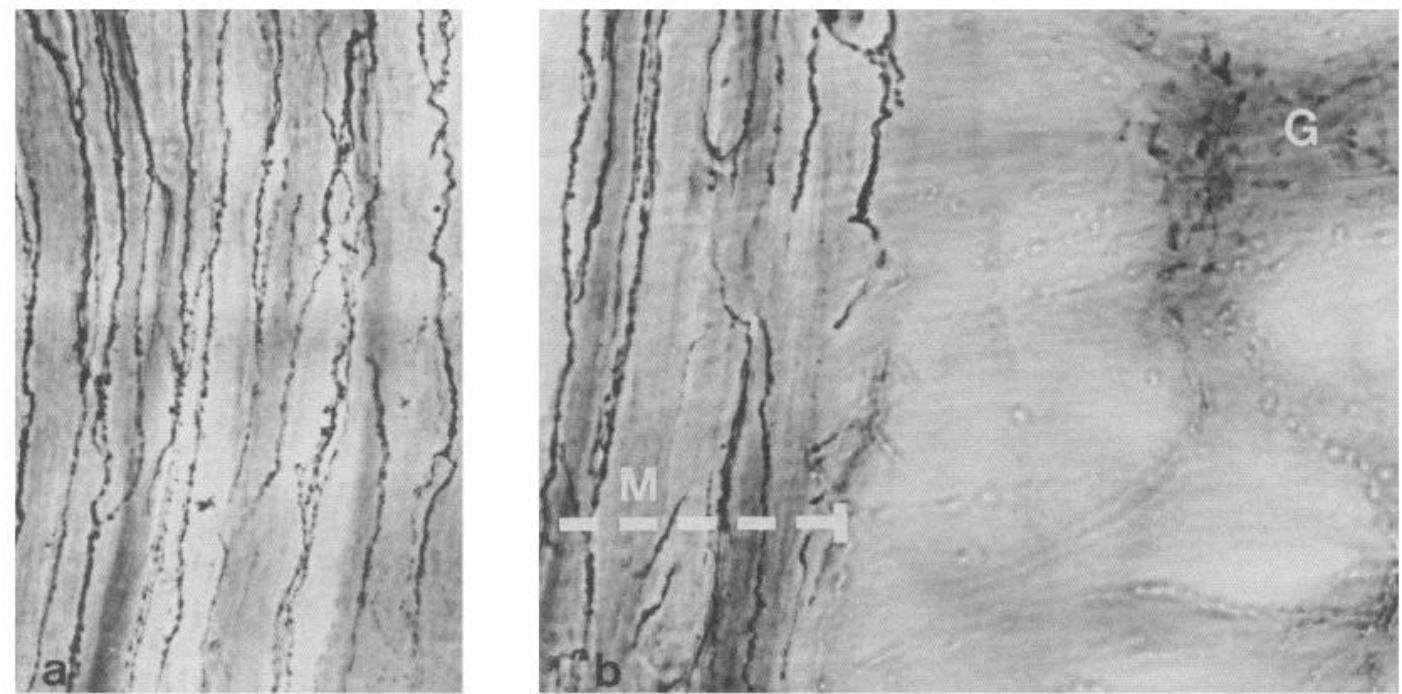

Figure 6. Immunoperoxidase micrographs of the guinea pig colon treated with anti-GABA antibodies. These micrographs illustrate the dense innervation of the circular muscle of the guinea pig colon by GABA immunopositive fibers. $a$, The typical dense, semiparallel arrangement of heavily immunopositive fibers. $b$, A band of circular muscle occupies the left-hand part of the field ( $M$, its extent indicated by a stippled line). A ganglion of myenteric plexus $(G)$ lies below the plane of focus, although immunopositive varicosities are discernible within it. Note the coarse appearance of the labeled fibers in the muscle when compared to those seen inside the primary myenteric plexus and in the tertiary plexus (see Fig. 5). $\times 400$.

inent site of GABA immunoreactivity in the present study. Heavily labeled fibers were observed within the myenteric ganglia, the interconnecting strands, and the circular muscle layer of both species studied; in the guinea pig, prominent label was also seen in the tertiary plexus. Although the possibility that some of these fibers originate outside the gut can be excluded only by denervation experiments, no evidence exists suggesting the presence of a GABAergic component in the extrinsic gut innervation. Similarily, we have as yet no evidence for GABAergic neuronal cell bodies in the submucous plexus, although this question has not yet been studied closely. It is therefore very likely that most of the GABA-containing axons seen in this study emanate from neuronal cell bodies within the myenteric plexus. The distribution of the labeled axons suggests that these neurons have at least two major projections: (1) to other neurons within the myenteric plexus and (2) to the circular muscle.

While projections within the myenteric plexus were seen in both the rat colon and in the guinea pig colon and ileum, they were most obvious in the rat colon. This plexus contained numerous intensely immunoreactive nerve fibers, some of which appeared to project for considerable distances along the longitudinal axis of the colon, while a dense fiber network was also seen among the neuronal cell bodies within the ganglia. A clue to the functional role of such intraganglionic fibers comes from intracellular recordings of single myenteric neurons. These studies have shown that a subpopulation of myenteric neurons, socalled AH cells, possesses GABA receptors and depolarizes on GABA application, while other neurons, the $S$ cells, are insensitive to GABA (Cherubini and North, 1984a). It was also found that GABA inhibits transmitter release from both cholinergic and noncholinergic terminals within myenteric ganglia (Cherubini and North, 1984b).

GABA-containing fibers were present within the circular muscle layer of both rat and guinea pig, although in the guinea pig the innervation was considerably denser. This observation is entirely consistent with our earlier finding, based on electronmicroscopic autoradiography, that some axons in intramuscular nerves of the guinea pig cecum selectively accumulate ${ }^{3} \mathrm{H}-\mathrm{GABA}$ (Jessen et al., 1983). Furthermore, it strongly supports our previous proposal, based on the autoradiographic experiments, that some myenteric GABAergic neurons project out from the myenteric ganglia, sending their axons into the intramuscular nerve bundles that characteristically form the presynaptic element of the gut neuromuscular junction (Jessen et al., 1983). The prominent immunohistochemical labeling of the tertiary plexus lying between the primary myenteric plexus and the adjacent circular muscle, described here in the guinea pig ileum and colon, constitutes additional evidence for a projection between the myenteric ganglia and the circular muscle. This arrangement, which on present evidence appears to be found in most of the guinea pig gastrointestinal tract, is intriguing in view of the well-confirmed observation that GABA has no direct action on gut muscle (Hobbiger, 1958; Kaplita et al., 1982; Krantis et al., 1980). The most likely explanation is that the functional role of GABAergic axons in intramuscular nerves is to act on neighboring axons to modulate presynaptically the release of transmitters, such as $\mathrm{ACh}$, which in turn act directly on the surrounding musculature (see Jessen et al., 1983).

This suggestion is consistent with morphological and pharmacological data. Ultrastructural studies have shown that the intramuscular nerves are a collection of often closely apposed varicose axons with a very loose Schwann cell ensheathment, clearly allowing for transmitter-mediated cross-talk between neighboring axons (Gabella, 1972). More recently, pharmacological studies have demonstrated that GABA could indeed act in this way. Applied GABA inhibits the cholinergic twitch induced by electrical nerve stimulation in the guinea pig ileum and also diminishes spontaneous release of the $\mathrm{ACh}$ from gut nerves (Bowery et al., 1981; Giotti et al., 1983; Kaplita et al., 1982; Krantis et al., 1980; Ong and Kerr, 1983). It has similar inhibitory effects on an electrically evoked non-cholinergic contractile response, possibly caused by release of substance $P$ (Ong and Kerr, 1983). In contrast to these inhibitory actions of GABA on active terminals, GABA causes release of ACh from resting cholinergic fibers in the gut, thereby indirectly causing muscle contraction (Kaplita et al., 1982; Kleinrok and Kilbinger, 1983; Krantis et al., 1980). Muscle relaxation is also sometimes observed in GABA application. This has been ascribed either to inhibition of tonic release of $\mathrm{ACh}$ or to stimulation of intrinsic inhibitory neurons (Giotti et al., 1983; Krantis et al., 1980). 
Although the pharmacological effects of GABA on the gut are complex and not fully understood, it is clear that GABA can affect transmitter release from enteric nerves. It remains to be determined whether this effect takes place exclusively within the myenteric plexus or, as we would predict, partly at the level of the terminal varicosities in the intramuscular nerves.

We conclude from the present work, and related observations, that a population of GABAergic neurons is intrinsic to the myenteric plexus. The projections of these cells ramify within myenteric ganglia and reach the terminal varicose axons of other neurons within the intramuscular nerves of the gut musculature. Electrophysiological studies indicate that the intraganglionic GABAergic fibers could have both pre- and postsynaptic actions, while in intramuscular nerves GABA is likely to act as a prejunctional modulator of enteric neuromuscular transmission. It is now important to unravel the precise functional role of these neurons within the complex neuronal circuitry of the gut wall.

\section{References}

Bowery, W. G., A. Doble, D. R. Hill, A. L. Hudson, J. S. Shaw, M. G. Turnbull, and R. Warrington (1981) Bicuculline-insensitive GABA receptors on peripheral autonomic nerve terminals. Eur. J. Pharmacol. 71: 53-70.

Cherubini, E., and R. A. North (1984a) Actions of $\gamma$-aminobutyric acid on neurones of guinea-pig myenteric plexus. Br. J. Pharmacol. 82: 93-100.

Cherubini, E., and R. A. North (1984b) Inhibition of calcium spikes and transmitter release by $\gamma$-aminobutyric acid in the guinea-pig myenteric plexus. Br. J. Pharmacol. 82: 101-105.

Cook, R. D., and G. Burnstock (1976) The ultrastructure of Auerbach's plexus in the guinea-pig. I. Neuronal elements. J. Neurocytol. 5: 171194.

Furness, J. B., and M. Costa (1980) Types of nerves in the enteric nervous system. Neuroscience 5: 1-20.

Furness, J. B., M. Costa, and J. R. Keast (1984) Choline acetyltransferase and peptide immunoreactivity of submucous neurons in the small intestine of the guinea pig. Cell Tissue Res. 237: 329-336.

Gabella, G. (1972) Innervation of the intestinal muscular coat. J. Neurocytol. $1:$ 341-362.

Giotti, A., S. Luzzi, S. Spagnesi, and L. Zilletti (1983) GABA and $\mathrm{GABA}_{\mathrm{B}}$ receptor mediated effects in guinea-pig ileum. Br. J. Pharmacol. 78: 469-478.

Hills, J. M., and K. R. Jessen (1983) Neuronal ${ }^{3}$ H-GABA release on field stimulation of an isolated gut preparation of guinea-pig taenia coli. J. Physiol. (Lond.) 334: 133P.

Hills, J. M., K. R. Jessen, and M. J. Saffrey (1985) Immunohistochemical evidence for GABAergic neurones in the enteric nervous system of the rat and guinea-pig. J. Physiol. (Lond.) 367: 55P.

Hobbiger, F. (1958) Effects of gamma-aminobutyric acid on the isolated mammalian ileum. J. Physiol. (Lond.) 142: 147-164.

Hodgson, A. J., B. Penke, A. Erdei, I. W. Chubb, and P. Somogyi (1985) Antisera to $\gamma$-aminobutyric acid. I. Production and characterization using a new model system. J. Histochem. Cytochem. 33: 229-239.

Jessen, K. R. (1981) GABA and the enteric nervous system. A neurotransmitter function? Mol. Cell. Biochem. 38: 69-76.

Jessen, K. R., R. Mirsky, M. E. Dennison, and G. Burnstock (1979) GABA may be a neurotransmitter in the vertebrate peripheral nervous system. Nature 281: 71-74.

Jessen, K. R., M. J. Saffrey, S. Van Noorden, S. R. Bloom, J. M. Polak, and G. Burnstock (1980) Immunohistochemical studies of the enteric nervous system in tissue culture and in situ: Localization of vasoactive intestinal polypeptide (VIP), substance-P and enkephalin immunoreactive nerves in the guinea-pig gut. Neuroscience 5: 17171735.

Jessen, K. R., J. M. Hills, M. E. Dennison, and R. Mirsky (1983) $\gamma$-Aminobutyrate as an autonomic neurotransmitter: Release and uptake of ${ }^{3} \mathrm{H}-\gamma$-aminobutyrate in guinea-pig large intestine and cultured enteric neurons using physiological methods and electron microscopic autoradiography. Neuroscience 10: 1427-1442.

Kaplita, P. V., D. H. Walters, and D. J. Triggle (1982) $\gamma$-Aminobutyric acid action on guinea-pig ileal myenteric plexus. Eur. J. Pharmacol. 79: 43-51.

Kerr, D. I. B., and A. Krantis (1983) Uptake and stimulus evoked release of ${ }^{3} \mathrm{H}-\gamma$-aminobutyric acid by mycnteric nervcs of guinea-pig intestine. Br. J. Pharmacol. 78: 271-276.

Kleinrok, A., and H. Kilbinger (1983) $\gamma$-Aminobutyric acid and cholinergic transmission in the guinea-pig ileum. Naunyn Schmiedebergs Arch. Pharmacol. 322: 216-220.

Krantis, A., and D. I. B. Kerr (1981) Autoradiographic localisation of ${ }^{3} \mathrm{H}$-gamma-aminobutyric acid in the myenteric plexus of the guineapig small intestine. Neurosci. Lett. 23: 263-268.

Krantis, A., M. Costa, J. B. Furness, and J. Orbach (1980) $\gamma$-Aminobutyric acid stimulates intrinsic inhibitory and excitatory nerves in the guinea-pig intestine. Eur. J. Pharmacol. 67: 461-468.

Legay, C., M. J. Saffrey, and G. Burnstock (1984) Coexistence of immunoreactive substance $P$ and serotonin in neurons of the gut. Brain Res. 302: 379-382.

Melander, T., T. Hökfelt, $\AA$. Rökaeus, J. Fahrenkrug, K. Tatemoto, and V. Mutt (1985) Distribution of galanin-like immunoreactivity in the gastrointestinal tract of several mammalian species. Cell Tissue Res. 239: 253-270.

Miki, Y., K. Taniyama, C. Tanaka, and T. Tobe (1983) GABA, glutamic acid decarboxylase and GABA transaminase levels in the myenteric plexus in the intestine of humans and other mammals. J. Neurochem. 40: 861-865.

Ong. J., and D. I. B. Kerr (1983) GABA and $_{A} \mathrm{GBA}_{\mathrm{B}}$-receptor mediated modification of intestinal motility. Eur. J. Pharmacol. 86: 917.

Pujol, J. F. (1984) Serotonin and GABA in Raphe neurons. In Coexistence of Neuroactive Substances in Neurons, V. Chan-Palay and S. L. Palay, eds., pp. 45-62, Wiley, New York.

Saffrey, M. J., N. Marcus, K. R. Jessen, and G. Burnstock (1983) Distribution of neurons with high affinity uptake sites for GABA in the myenteric plexus of the guinea-pig, rat and chicken. Cell Tissue Res. 234: 231-235.

Schultzberg, M., T. Hökfelt, G. Nilsson, L. Terenius, J. F. Rehfeld, M. Brown, R. Elde, M. Goldstein, and S. Said (1980) Distribution of peptide- and catecholamine-containing neurons in the gastrointestinal tract of rat and guinea-pig: Immunohistochemical studies with antisera to substance $P$, vasoactive intestinal polypeptide, enkephalins, somatostatin, gastrin, cholecystokinin, neurotensin and dopamine $\beta$-hydroxylase. Neuroscience 5: 689-744.

Somogyi, P., and A. J. Hodgson (1985) Antisera to $\gamma$-aminobutyric acid. III. Demonstration of GABA in Golgi-impregnated neurons and in conventional electron microscopic sections of cat striate cortex. J. Histochem. Cytochem. 33: 249-257.

Somogyi, P., A. J. Hodgson, A. D. Smith, N. G. Nunzi, A. Gorio, and J.-Y. Wu (1984) Different populations of GABAergic neurons in the visual cortex and hippocampus of cat contain somatostatin- or cholecystokinin-immunoreactive material. J. Neurosci. 4: 2590-2603.

Somogyi, P., A. J. Hodgson, I. W. Chubb, B. Penke, and A. Erdei (1985) Antisera to $\gamma$-aminobutyric acid. II. Immunocytochemical application to the central nervous system. J. Histochem. Cytochem. 33:240248.

Tanaka, C., and K. Taniyama (1985) Substance P provoked $\gamma$-aminobutyric acid release from the myenteric plexus of the guinea-pig small intestine. J. Physiol. (Lond.) 362: 319-329.

Taniyama, K., M. Kusunoki, N. Saito, and C. Tanaka (1982a) Release of $\gamma$-aminobutyric acid from cat colon. Science 217: 1038-1040.

Taniyama, K., Y. Miki, and C. Tanaka (1982b) Presence of $\gamma$-aminobutyric acid and glutamic acid decarboxylase in Auerbach's plexus of cat colon. Neurosci. Lett. 29: 53-56.

Taniyama, K., Y. Miki, M. Kusunoki, N. Saito, and C. Tanaka (1983) Release of endogenous and labeled GABA from isolated guinea-pig ileum. Am. J. Physiol. 245: G717-G721.

Wolter, H. J. (1985) $\alpha$-Melanotropin, $\beta$-endorphin and adrenocorticotropin-like immunoreactivities are localized within duodenal myenteric plexus perikarya. Brain Res. 325: 290-293. 